\title{
TURBULENCE IN THE ORION NEBULA
}

\author{
H.O. Castañeda ${ }^{1}$ and C.R. O'Dell ${ }^{2}$ \\ ${ }^{1}$ Inst. de Astrofisica de Canarias, Spain \\ ${ }^{2}$ Rice University, USA
}

We have examined the radial and turbulent velocities of the Orion Nebula at the seeing limit, observing the $[\mathrm{OIII}] \lambda 5007$ line and the $[\mathrm{OII}] \lambda 3727$ doublet with the coudé feed system of the Kitt Peak National Observatory, at very high spatial and spectral resolution.

We show evidence for the existence of multiple components in the nebular profile of the different lines, representing different elements of the gas flow. We find at least four distinct flows, including an highly blueshifted [OII] component over the entire observed area. A model to explain the velocity structure is advanced, as the interaction of a "champagne" flow with a stellar wind bubble and secondary streams from inhomogeneities in the nebula.

The structure function has been calculated and compared with the Kolmogorov theory of turbulence. There is evidence for a correlation between random velocities and distance across the face of the nebula, but the observation are in disagreement with the classical theory for nebular turbulence.

\section{HIGH-RESOLUTION MOLECULAR LINE OBSERVATIONS OF THE CORE AND OUTFLOW IN ORION B}

\author{
J.S. Richer, R.E. Hills and R. Padman \\ Mullard Radio Astronomy Observatory, Cambridge, England
}

\begin{abstract}
High-resolution CO $J \rightarrow 1 \rightarrow 2$ observations of the Orion B molecular outflow show that the outflow is unipolar, and that there is evidence of acceleration of molecular gas at up to $0.5 \mathrm{pc}$ from the driving star. The highest-velocity material, as well as being furthest from the source, seems to lie close to the central axis of the flow, and is presumably being accelerated by entrainment in the flow or jet emanating from the star. We have also mapped the $\mathrm{HCO}^{+}$ $J=3 \rightarrow 2$ emission at 19 -arcsec resolution. We derive an excitation temperature of around 25 $\mathrm{K}$ in the cloud core, and a core mass of about $75 M_{\odot}$, this estimate is in accord with a model in which the core has a kinetic temperature of $30-50 \mathrm{~K}$, with no molecular depletion on to grains. This is in contrast to the recent suggestion that the core contains cold isothermal protostars.
\end{abstract}

